\title{
An investigation into the effect of artificially sweetened beverage consumption on dietary intake in a population of UK adults
}

\author{
A. Oslizlok and S. Drummond \\ School of Health Sciences, Queen Margaret University, Edinburgh EH21 6UU
}

Obesity has reached epidemic proportions in Scotland with $62 \%$ of the population reported as overweight or obese ${ }^{(1)}$. Sugar sweetened beverages (SSB's) have previously been associated with weight gain ${ }^{(2,3)}$, prompting many to switch to lower-calorie artificially sweetened beverages (ASB's). Evidence that artificial sweeteners are effective in weight management is equivocal and recent epidemiological studies suggest AS usage may in fact be associated with long term weight gain ${ }^{(4,5)}$. This study investigated the effect of substitution of SSB's with ASB's on dietary intake in a sample of habitual SSB consumers. Alterations to total energy intakes, carbohydrate, sugar, non-milk extrinsic sugar (NMES), fat and protein following substitution with ASB were measured. Pattern of beverage consumption and lifestyle characteristics were also explored to identify any significant associations (data not presented here).

In this two week intervention trial, week 1 acted as the control week when subjects $(n=17)$ were instructed to record their normal dietary intake for three consecutive days, maintaining usual SSB consumption. In week 2, subjects substituted their usual SSB's for the artificially sweetened alternative and again recorded dietary intake for three days. Food diaries were analysed using WinDiets software (2005) and mean intakes of energy and macronutrients between control and intervention weeks compared to identify any effect of beverage substitution.

Substitution of SSB's with ASB's resulted in significant reductions in mean energy $(1055 \pm 1412 \mathrm{~kJ})$, total carbohydrate (72.1 $\pm 11.8 \mathrm{~g})$, sugar $(70.7 \pm 30.1 \mathrm{~g})$ and NMES $(73 \pm 29.6 \mathrm{~g})$ intakes. Absolute intakes of protein and fat (grams) did not differ significantly as a result of the intervention $(p>0.05$ for both), however the $\%$ energy provided by both nutrients increased significantly $(+1.9 \pm 2.6 \%$ for protein, $+7.9 \pm 11.5 \%$ for fat). A significant inverse relationship was observed between $\%$ energy provided by sugar and fat both at baseline $(r=-0.5, p<0.05)$, and for the total duration of the 6-day study period $(r=-0.91, p<0.05)$, but there was no evidence of a significant relationship between sugar and fat during the intervention week, either between total intakes $(r=0.1, p>0.05)$ or $\%$ energy provided by both nutrients $(r=-0.4, p>0.05)$.

Direct substitution of SSB's for ASB's results in a short term reduction of total energy, carbohydrate, total sugar and NMES intake, with no evidence of immediate caloric compensation elsewhere in the diet. ASB's may therefore be beneficial in preventing excess weight gain, if consumed as part of a multidisciplinary weight management program. The long term effects of ASB's on dietary intake and weight status are still unclear and further research on the topic is required.

1. Scottish Health Survey (2009) Available at: www.scotland.gov.uk/Publications

2. James J, Thomas P, Cavan D \& Kerr D (2004) Brit Med J, 328; 1237.

3. Malik VS, Schulze MB \& Hu FB (2006) Am J Clin Nutr 84 (2); 274-288.

4. Fowler SP, Williams K, Resendez RG, Hunt KJ, Hazuda HP \& Stern MP (2008) Obesity 16; 1894-1900.

5. Swithers SE, Martin AA \& Davidson TL (2010) Physiol \& Behav 100 (1); 55-62. 